\title{
Composite Learning Sliding Mode Control of Nonlinear Systems Subject to Actuator Failures
}

\author{
Xianmin Hou $(\mathbb{D})$ and Songyu Wang \\ School of Information and Management Sciences, Henan Agricultural University, Zhengzhou 450046, China \\ Correspondence should be addressed to Xianmin Hou; houxianmin@henau.edu.cn
}

Received 7 November 2020; Revised 22 November 2020; Accepted 25 November 2020; Published 7 December 2020

Academic Editor: Heng Liu

Copyright (c) 2020 Xianmin Hou and Songyu Wang. This is an open access article distributed under the Creative Commons Attribution License, which permits unrestricted use, distribution, and reproduction in any medium, provided the original work is properly cited.

\begin{abstract}
This article focuses on controlling single-input-single-output (SISO) nonlinear systems with actuator failures via sliding mode control (SMC) and composite learning SMC (CLSMC). In the design of the SMC, an integer-order sliding surface is proposed, and an adaptive law is constructed to update the parameter evaluation in the actuator failure. The SMC method can achieve the tracking error approaching zero if a strict permanent excitation (PE) condition is satisfied. To mitigate this requirement, by using all data recorded while the controller works, we construct prediction errors that are utilized to produce a composite learning adaptive law. Then, the proposed CLSMC method not only drives the tracking error to zero but also realizes the accurate evaluation of the unmatched unknown parameter in the actuator failure. In addition, in the proposed CLSMC method, we only need to satisfy an interval excitation (IE) condition. Simulation results are presented to indicate the validity of our methods.
\end{abstract}

\section{Introduction}

It is commonly recognized that actuator failures in the control of nonlinear systems usually makes the control process more complicated and reduces the control performance. To deal with this problem, fault-tolerant control (FTC) was proposed, for example, an FTC method was proposed for SISO system with actuator failures in [1], where only matched system uncertainty is taken into consideration. For unknown SISO systems, adaptive fuzzy FTC (AFFTC) methods were introduced in $[2,3]$, where fuzzy systems are used to model the systems. By using a disturbance observer, an adaptive decentralized FTC of large-scale systems subject to actuator failures was addressed in [4], which considers two types of actuator failures, i.e., loss of effectiveness type and stuck type. In addition, different actuator failures, for example, loss of effectiveness and bias are presented in [5]. More meaningful results about FTC can be seen in [6-9]. It is also well known that the sliding mode control (SMC) method is also been proposed for nonlinear systems with actuator faults. For example, the SMC for nonlinear systems with actuator faults via disturbance observer was studied in [10]. Fuzzy adaptive SMC for MIMO nonlinear systems was studied in [11]. A novel sliding surface was introduced to handle the problem of multiple inputs containing sector nonlinearities and dead-zones in [12]. Although FTC of nonlinear systems has been studied by many scientists and fruitful results has been produced, in above literature, unmatched parametric uncertainties are not taken into account.

In the adaptive control, one staple objective is to guarantee the convergence of parameters. However, people often need accurate estimations of unknown parameters. Therefore, to accelerate the convergence speed and achieve accurate estimation of an unknown parameter, composite adaptive control (CAC) was introduced by combining tracking error and prediction error in [13]. In [14], an antidisturbance method was given for nonlinear systems with multisource disturbances by using an adaptive disturbance observer. In [15], a CAC method for fractionalorder systems was proposed via fractional dynamic surface and backstepping technique. Composite neural network control for fractional-order system with actuator failures was presented in [16], where two types of failures are considered 
and a fractional filter is also implemented to solve the "explosion of terms" problem in the backstepping controller design. However, in above literature, a strict condition, i.e., the permanent excitation (PE), should be satisfied to guarantee the convergence of the adaptive parameters. To relax this limitation, a more powerful control method called composite learning control (CLC) was proposed in [17], where only a condition named interval excitation (IE) must be satisfied. In fact, the IE is much weaker than PE and easier to be satisfied in real-world systems. In the CLC method, all the data in the control process are recorded and used to produce a prediction error. Then, both the prediction error and the instantaneous tracking error are used to construct a composite learning law. Up to now, CLC has been investigated by some researchers. In [18], by using CLC in adaptive neural network control, the unknown control objective can be modeled accurately. In [19], CLC for MIMO systems was investigated, where the actual application of this method in the control of robotics is also considered. In [20], the CLC method was used to control fractional-order systems, where a fractional sliding surface is designed. Some other interesting CLC methods can be referred to [21-23]. However, to the best of our knowledge, the CLC for nonlinear systems with actuator faults has not been investigated.

From the above discussion, in this work, we study the SMC and the composite learning SMC (CLSMC) for nonlinear systems with actuator failures. The SMC with adaptive law is designed to ensure the convergence of tracking error but cannot accurately evaluate the parameter. In order to obtain accurate parameter evaluation, the CLSMC method with composite learning adaptive law is designed. The contributions to this article are as follows: (1) a sliding surface is designed for strict-feedback nonlinear systems to facilitate the CLC design; (2) actuator failures with mismatched parameters are discussed, and a CLSMC method is proposed to obtain the accurate estimation of parametric uncertainties under the IE condition.

The structure of this work is as follows. In Section 2, the problem statement, actuator failures model, the SMC method, and the CLSMC method are presented. Section 3 gives a simulation example. Finally, Section 4 summarizes this work.

\section{Construction of SMC and CLSMC}

2.1. Problem Description. Consider the SISO nonlinear systems as

$$
\left\{\begin{array}{l}
\dot{x}_{i}(t)=x_{i+1}(t), \\
\dot{x}_{n}(t)=f(x(t))+\sum_{k=1}^{q} u_{k}(t),
\end{array}\right.
$$

where $i=1,2, \ldots, n-1, x(t)=\left[x_{1}(t), \ldots, x_{n}(t)\right]^{T} \in \mathscr{R}^{n}$ is the state vector, $f(x(t)): \mathscr{R}^{n} \mapsto R$ are known continuous nonlinear functions, and $u_{k}(t), k=1, \ldots, q$ is the actuator's output. In this paper, $k$ control inputs are considered, where some inputs subject to actuator faults are listed as follows.

There are two types of the actuator failures to be discussed here. One is the stuck type which is described as

$$
u_{k}(t)=\widetilde{u}_{k}(t)=\widetilde{u}_{k}(t)+\phi_{k}^{T}(x(t)) \theta, \quad t \geq T_{k},
$$

with $k \in\left\{k_{1}, \ldots, k_{p}\right\} \subset\{1, \ldots, q\}, p \in \mathcal{N}, p \leq q-1, \widetilde{u}_{k}$ being a constant, $T_{k}$ being the time when failure occurs, $\phi_{k}(x(t)): \mathscr{R}^{n} \mapsto \mathscr{R}^{l}$ with $l \in \mathcal{N}$ being a function, and $\theta=$ $\left[\theta_{1}, \ldots, \theta_{l}\right]^{T} \in \mathscr{R}^{l}$ being an unknown constant vector.

Another is the loss of effectiveness type which is described as

$$
u_{k}(t)=\varrho_{k} v_{k}(t), \quad t>T_{k},
$$

with $0<\varrho_{k} \leq 1, k \in \overline{\left\{k_{1}, \ldots, k_{p}\right\}} \subset\{1, \ldots, q\}, \varrho_{k}$ being a known parameter, and $v_{k}(t)$ is the control input.

The actuator failures are defined as follows:

$$
\begin{aligned}
u_{k}(t) & =\delta_{k} \breve{u}_{k}(t)+\left(1-\delta_{k}\right) \varrho_{k} v_{k}(t), \\
\delta_{k} & = \begin{cases}1, & u_{k}(t)=\breve{u}_{k}(t), \\
0, & u_{k}(t) \neq \breve{u}_{k}(t) .\end{cases}
\end{aligned}
$$

Remark 1. In this paper, two kinds of input faults are considered. Consider the stuck type (2), the failure type (3), and the actuator failures (4). In (4), when $\delta_{k}=0$, (4) becomes the failure type where $\widetilde{u}_{k}=0, \phi_{k}^{T}(x(t))=\mathbf{0}$. When $\delta_{k}=1$, (4) means the stuck type, where $v_{k}(t)=0$ for $t>T_{k}$.

Assuming the ideal signal is $x_{d}(t)=\left[x_{d 1}(t), \ldots\right.$, $\left.x_{d n}(t)\right] \in \mathscr{R}^{n}$, it is a smooth function where $x_{d}(t)$ and $\dot{x}_{d}(t)$ are of $\mathscr{L}_{\infty}$ and $\dot{x}_{d i}(t)=x_{d(i+1)}(t)$ for $i=1,2, \ldots, n-1$. The tracking error is designed as

$$
e(t)=x(t)-x_{d}(t)
$$

and the parameter evaluation error is designed as

$$
\widetilde{\theta}(t)=\widehat{\theta}(t)-\theta
$$

where $\hat{\theta}(t)$ represents the estimation of $\theta$.

Here are some definitions and assumptions we will use in the following article.

Definition 1. $\phi(t)$ is the IE in the interval $\left[T-\sigma_{0}, T\right]$ with $0<\sigma_{0}<T$ when $\phi(t)$ meet $\int_{T-\sigma_{0}}^{T} \phi^{T}(\sigma) \phi(\sigma) \mathrm{d} \sigma \geq \iota I_{m \times m}$ with $\iota \in \mathscr{R}^{+}$

Definition 2. $\phi(t)$ is the $\mathrm{PE}$ when $\phi(t)$ meet $\int_{t-\sigma_{0}}^{t} \phi^{T}(\sigma) \phi(\sigma) \mathrm{d} \sigma \geq \iota I_{m \times m}$ with $\iota \in \mathscr{R}^{+}, \sigma_{0}>0$, and $t \in \mathscr{R}^{+}$.

Assumption 1. Atmost $q-1$ control input fault is (2), and the remaining fault is (3), SISO nonlinear system (1) can also achieve the control goal.

2.2. Tradition SMC Design. Next, we will introduce an integer-order sliding surface, which is constructed as

$$
S(t)=\sum_{i=1}^{n} \rho_{i} e_{i}(t),
$$


where $\rho_{i}$ in this formula is chosen to make $S(t)$ converge as faster as possible. From formula (7), we can get the following formula:

$$
\begin{aligned}
\dot{S}(t) & =\sum_{i=1}^{n} \rho_{i} \dot{e}_{i}(t), \\
& =\left[\begin{array}{lll}
\rho_{1}, & \rho_{2}, \ldots & \rho_{n}
\end{array}\right]\left[\begin{array}{c}
\dot{e}_{1}(t) \\
\dot{e}_{2}(t) \\
\vdots \\
\dot{e}_{n}(t)
\end{array}\right], \\
& =\left[\begin{array}{c}
\rho_{1}, \rho_{2}, \ldots \rho_{n}
\end{array}\right]\left[\begin{array}{c}
x_{2}(t)-\dot{x}_{d 1}(t) \\
x_{3}(t)-\dot{x}_{d 2}(t) \\
\vdots \\
f(x(t))+\sum_{k=1}^{q} u_{k}(t)-\dot{x}_{d n}(t)
\end{array}\right], \\
& =\sum_{i=1}^{n-1} \rho_{i} x_{i+1}(t)-\sum_{i=1}^{n} \rho_{i} \dot{x}_{d i}(t)+\rho_{n}\left[f(x(t))+\sum_{k=1}^{q} u_{k}(t)\right] .
\end{aligned}
$$

We construct the control input as

$$
v_{k}(t)=c_{k 1} v^{*}(t)+c_{k 2},
$$

where $v^{*}(t)$ is the real controller and $c_{k 1}$ and $c_{k 2}$ are positive constants that satisfy

$$
\begin{aligned}
& \sum_{k=1}^{q}\left(1-\delta_{k}\right) \varrho_{k} c_{k 1}=1, \\
& \sum_{k=1}^{q}\left(1-\delta_{k}\right) \varrho_{k} c_{k 2}=-\sum_{k=1}^{q} \delta_{k} \widetilde{u}_{k} .
\end{aligned}
$$

From (4), (8), and (9), we get the following:

$$
\begin{aligned}
\dot{S}(t)= & \sum_{i=1}^{n-1} \rho_{i} x_{i+1}(t)-\sum_{i=1}^{n} \rho_{i} \dot{x}_{d i}(t)+\rho_{n} f(x(t)) \\
& +\rho_{n} \sum_{k=1}^{q}\left[\delta_{k} \breve{u}_{k}(t)+\left(1-\delta_{k}\right) \varrho_{k} v_{k}(t)\right], \\
= & \sum_{i=1}^{n-1} \rho_{i} x_{i+1}(t)-\sum_{i=1}^{n} \rho_{i} \dot{x}_{d i}(t)+\rho_{n} f(x(t)) \\
& +\rho_{n} v^{*}(t) \sum_{k=1}^{q}\left(1-\delta_{k}\right) \varrho_{k} c_{k 1}+\rho_{n} \sum_{k=1}^{q} \delta_{k} \widetilde{u}_{k} \\
& +\rho_{n} \sum_{k=1}^{q} \delta_{k} \phi_{k}^{T}(x(t)) \theta+\rho_{n} \sum_{k=1}^{q}\left(1-\delta_{k}\right) \varrho_{k} c_{k 2} .
\end{aligned}
$$

Based on (10) and (11), the above expression is written as

$$
\begin{aligned}
\dot{S}(t)= & \sum_{i=1}^{n-1} \rho_{i} x_{i+1}(t)-\sum_{i=1}^{n} \rho_{i} \dot{x}_{d i}(t) \\
& +\rho_{n}\left[f(x(t))+v^{*}(t)+\sum_{k=1}^{q} \delta_{k} \phi_{k}^{T}(x(t)) \theta\right] .
\end{aligned}
$$

Make $v^{*}(t)$ as

$$
\begin{aligned}
v^{*}(t)= & -f(x(t))-\sum_{k=1}^{q} \delta_{k} \phi_{k}^{T}(x(t)) \hat{\theta}(t) \\
& +\frac{1}{\rho_{n}}\left[\sum_{i=1}^{n} \rho_{i} \dot{x}_{d i}(t)-\sum_{i=1}^{n-1} \rho_{i} x_{i+1}(t)-b S(t)\right] .
\end{aligned}
$$

The adaptive law is constructed as

$$
\left\{\begin{array}{l}
\Upsilon(t)=\varpi \sum_{k=1}^{q} \delta_{k} \phi_{k}(x(t)) S(t), \\
\dot{\hat{\theta}}(t)=\Omega(\hat{\theta}(t), \Upsilon(t)),
\end{array}\right.
$$

with $₫$ being a positive parameter, and $\Omega(\hat{\theta}(t) t, n \Upsilon q(t))$ is constructed as

$$
\Omega(\hat{\theta}(t), \Upsilon(t))= \begin{cases}\Upsilon(t), & \text { if }\|\hat{\theta}(t)\| \leq \alpha, \\ \Upsilon(t)-\frac{\widehat{\theta}(t) \hat{\theta}^{T}(t) \Upsilon(t)}{\|\widehat{\theta}(t)\|^{2}}, & \text { otherwise, }\end{cases}
$$

where $\alpha>0$

Remark 2. It should be mentioned that the proposed adaptation law (16) has some applications. Firstly, it can obtain the objective of accurate estimation of the unknown parameter $\theta$. Secondly, the boundedness of the updated parameter can be guaranteed by introducing a term $\alpha$. In fact, if $\|\theta(t)\|$ is too large, i.e., $\|\widehat{\theta}(t)\| \geq \alpha$, then the second equation in (16) is activated, and a negative term $-\left(\hat{\theta}(t) \widehat{\theta}^{T}(t)\right.$ $\Upsilon(t)) /\|\widehat{\theta}(t)\|^{2}$ will drive $\theta$ convergence rapidly.

Theorem 1. For SISO nonlinear systems satisfying Assumption 1, the control input (14) and the adaptive law (15) ensures that all signals are bounded and $e(t)$ approaches 0 .

Proof. Substituting (14) into (13) yields

$$
\dot{S}(t)=-b S(t)-\rho_{n} \sum_{k=1}^{q} \delta_{k} \phi_{k}^{T}(x(t)) \tilde{\theta}(t) .
$$

Let the Lyapunov function be

$$
\mathscr{V}(t)=\frac{1}{2} S^{2}(t)+\frac{\rho_{n}}{2 \varpi} \widetilde{\theta}^{T}(t) \widetilde{\theta}(t) .
$$

Differentiating (18) along (15) and (17) gives 


$$
\dot{\mathscr{V}}(t)=-b S^{2}(t)-\rho_{n} \widetilde{\theta}^{T}(t)\left[\sum_{k=1}^{q} \delta_{k} \phi_{k}(x(t)) S(t)-\frac{\dot{\hat{\theta}}(t)}{\emptyset}\right] .
$$

Substituting (15) into (19) yields

$$
\dot{\mathscr{V}}(t) \leq-b S^{2}(t)
$$

That means the system is asymptotically stable, and this ends the proof of Theorem 1 .

2.3. CLSMC Design. From the tradition SMC method above, we can see that the convergence of $e(t)$ can be guaranteed under the PE condition. Next, we will introduce a CLSMC method to ensure $e(t)^{\prime}$ s convergence and $\theta$ 's accurate evaluation in the absent of the PE condition.

Let $\phi_{k}^{T}(x(t))$ be an IE in $\left[T-\sigma_{0}, T\right]$ and the prediction evaluation error is

$$
\xi(t)=\chi(x(t)) \tilde{\theta}(t)
$$

with $\chi(x(t)): \mathscr{R}^{n} \mapsto R^{z \times z}$ being constructed as

$$
\chi(x(t))= \begin{cases}\mathbf{0}_{z \times z}, & \text { for } t \leq \sigma_{0}, \\ \int_{t-\sigma_{0}}^{t} \Psi(x(\sigma)) \Psi^{T}(x(\sigma)) \mathrm{d} \sigma, & \text { for } t>\sigma_{0},\end{cases}
$$

with $\Psi(x(t))=\rho_{n} \sum_{k=1}^{q} \delta_{k} \phi_{k}(x(t))$.

The composite learning adaptive law is constructed as

$$
\left\{\begin{array}{l}
\Upsilon(t)=\omega\left[\sum_{k=1}^{q} \delta_{k} \phi_{k}(x(t)) S(t)-\gamma \xi(t)\right], \\
\dot{\hat{\theta}}(t)=\Omega(\hat{\theta}(t), \Upsilon(t)),
\end{array}\right.
$$

with $\gamma>0$, and definition of $\Omega(\hat{\theta}(t) t, n \Upsilon q(t))$ is the same as that of (16).

The next task is to compute $\xi(t)$, in order to accomplish this task, we define

$$
\vartheta(t)=\int_{t-\sigma_{0}}^{t} \Psi(x(\sigma)) \Psi^{T}(x(\sigma)) \theta \mathrm{d} \sigma .
$$

Multiply both ends of (17) by $\Psi(x(t))$

$\Psi(x(t)) \Psi^{T}(x(t)) \theta=\Psi(x(t))\left[\dot{S}(t)+b S(t)+\Psi^{T}(x(t)) \hat{\theta}(t)\right]$.

From (24) and (25), we get

$\vartheta(t)=\int_{t-\sigma_{0}}^{t} \Psi(x(\sigma))\left[\dot{S}(\sigma)+b S(\sigma)+\Psi^{T}(x(\sigma)) \hat{\theta}(\sigma)\right] \mathrm{d} \sigma$.

From the above equation, we can get

$$
\begin{aligned}
\xi(t)= & \chi(x(t)) \hat{\theta}(t)-\int_{t-\sigma_{0}}^{t} \Psi(x(\sigma))[\dot{S}(\sigma)+b S(\sigma) \\
& \left.+\Psi^{T}(x(\sigma)) \hat{\theta}(\sigma)\right] \mathrm{d} \sigma .
\end{aligned}
$$

Remark 3. From the above discussion, we can get the algorithm of $\xi(t)$. So, by Definition 1, we can see that if $\chi(x(t)) \leq \iota$, then $\xi(t)=0$. Conversely, if $\chi(x(t))>\iota I$, then we have

$$
\xi(t)=\chi(x(t)) \hat{\theta}(t)-\vartheta(t) .
$$

In addition, we can see from formula (26) that, in order to figure out the value of $\vartheta(t)$, we have to know the value of $\dot{S}(\sigma)$. Because the value of $\dot{S}(\sigma)$ cannot be obtained directly, but we can get all the data of $S(t)$ that will help us to get to $\dot{S}(t)$.

Theorem 2. For SISO nonlinear systems satisfying Assumption 1, the control input (14) and the composite learning adaptive law (23) ensure that $\tilde{\theta}$ and $e(t)$ approaches 0.

Proof. Let the Lyapunov function and its derivatives be the same as (18) and (19). Substituting (23) into (19) gives

$$
\begin{aligned}
& \dot{\mathscr{V}}(t) \leq-b S^{2}(t)-\gamma \rho_{n} \tilde{\theta}^{T}(t) \xi(t), \\
& =-b S^{2}(t)-\gamma \rho_{n} \tilde{\theta}^{T}(t)[\chi(x(t)) \tilde{\theta}(t)-\vartheta(t)], \\
& =-b S^{2}(t)-\gamma \rho_{n} \tilde{\theta}^{T}(t) \chi(x(t)) \widetilde{\theta}(t), \\
& \leq-b S^{2}(t)-\gamma \iota \rho_{n} \tilde{\theta}^{T}(t) \tilde{\theta}(t), \\
& \leq-\mathrm{d} \mathscr{V}(t),
\end{aligned}
$$

with $d=\min \{2 b, 2 \varpi \iota \gamma\}$. So, the unknown parameter $\tilde{\theta}$ and $e(t)$ approaches 0 , and this ends the proof of Theorem 2 .

Remark 4. In [24], the concurrent learning control methods are used to get accurate parameter evaluation without the $\mathrm{PE}$ condition. The difference between this method and the introduced composite learning method is the usage of online recording data. The prediction error of concurrent learning control method is produced by recording data. Furthermore, prediction error is applied to get the accurate parameter evaluation under the IE condition, so sufficient online recording data are used. From the above discussion, it can be seen that the use of concurrent learning control method will add a lot of computing burden to the system. However, the introduced CLSMC method can reduce the computation because the online recording data can be obtained through (22) and (24), and maximizing the singular values is required. In addition, the use of integral can also reduce the measurement deviation of $\vartheta$.

Remark 5. It should be emphasized that the SMC method and the CLSMC method use the same control input (15), both of which can make $e(t)$ approaches 0 . The difference between the two methods lies in the CLSMC method which uses the composite learning adaptive law (23) to obtain accurate parameter evaluation. However, the SMC method does not have the ability to do that it can only make $\hat{\theta}(t)$ bounded. Therefore, it can be concluded that the CLSMC method has better control ability, which can also be seen from the simulation results. 


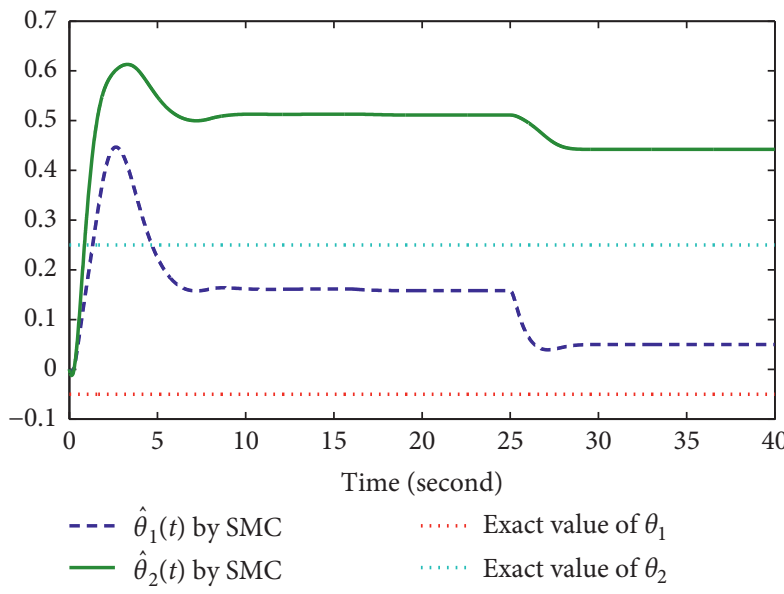

(a)

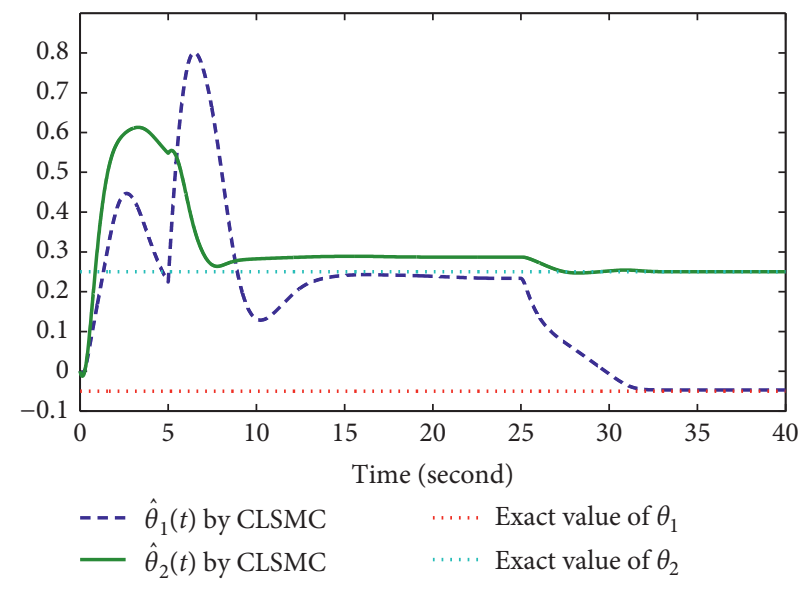

(b)

FIgURE 1: Parameter evaluations under SMC and CLSMC.

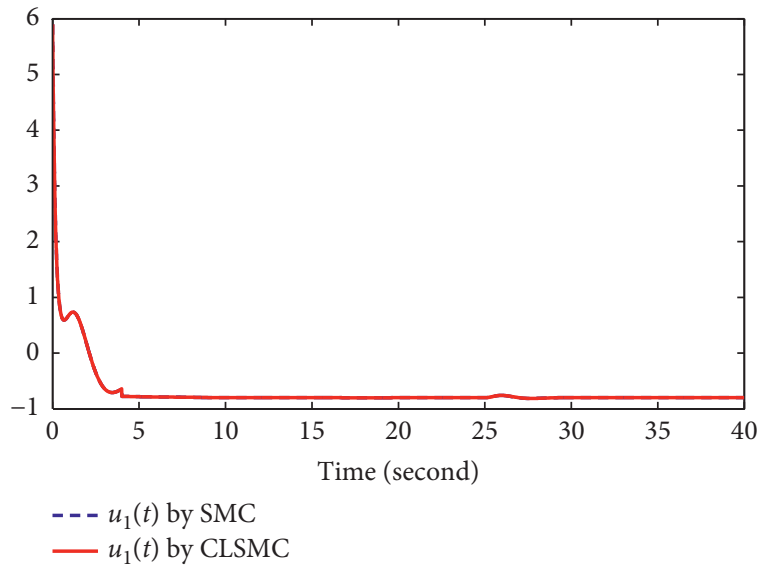

(a)

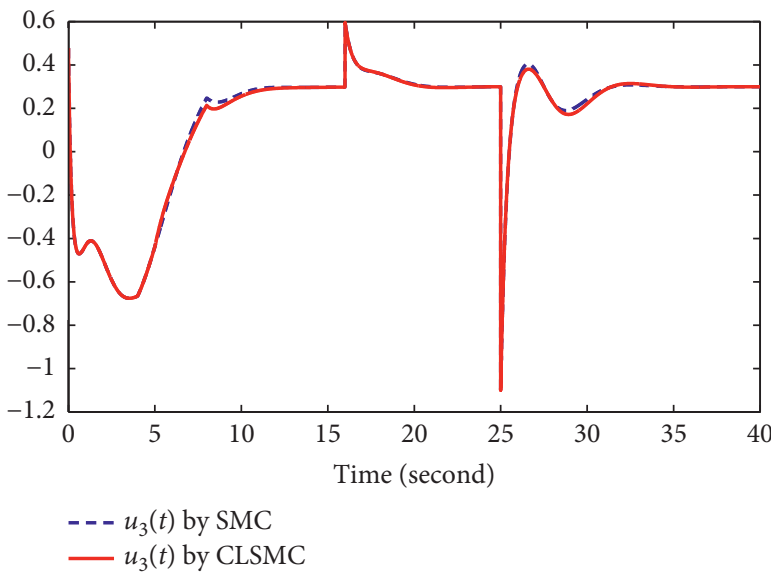

(c)

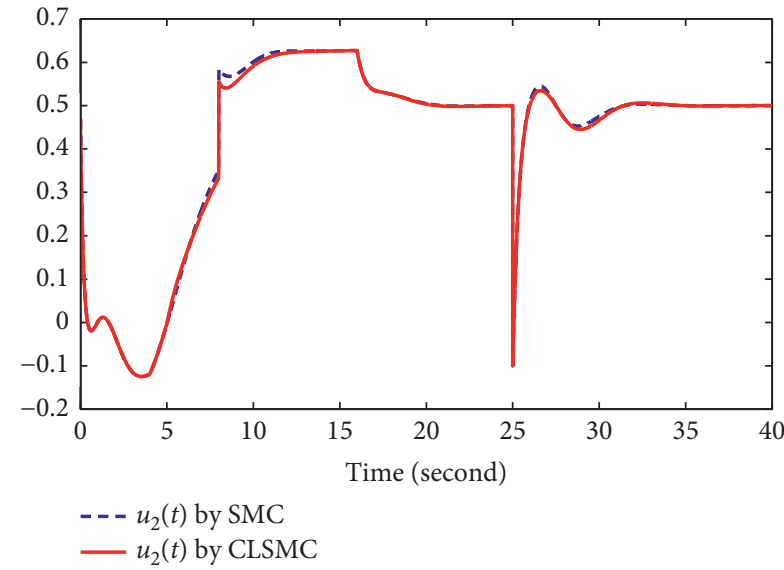

(b)

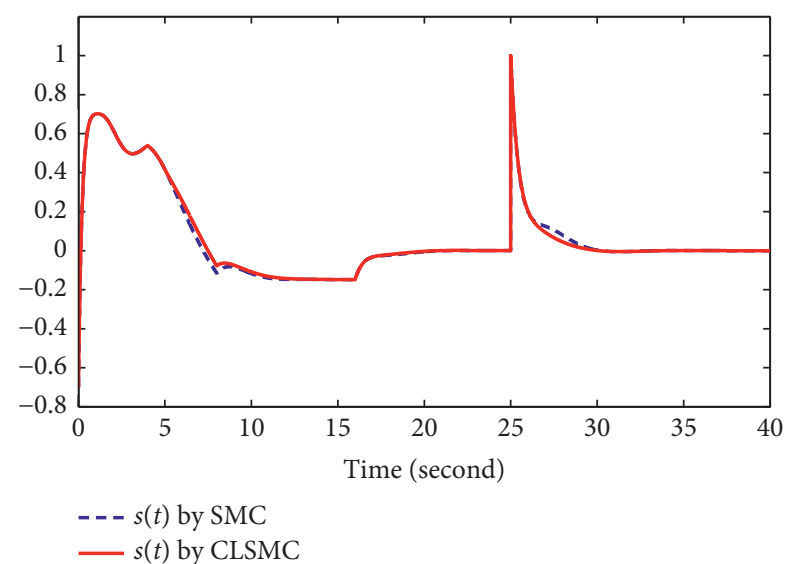

(d)

Figure 2: Control inputs and sliding surface of SMC and CLSMC. 


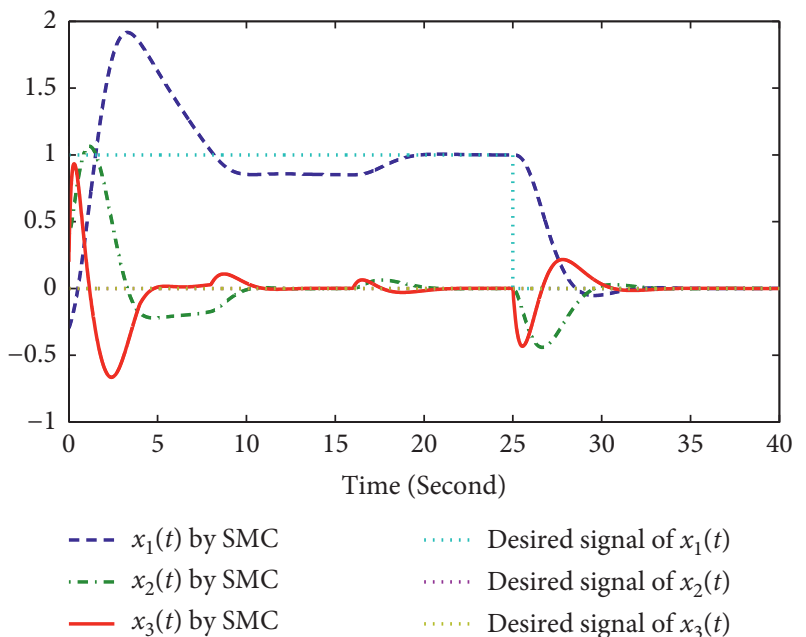

(a)
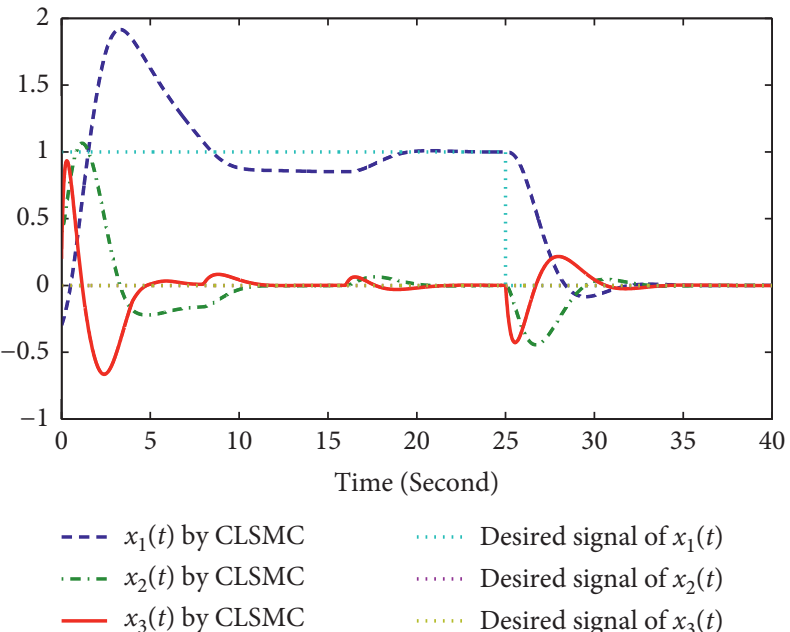

Desired signal of $x_{1}(t)$

,..... Desired signal of $x_{2}(t)$

Desired signal of $x_{3}(t)$

(b)

Figure 3: State variables of SMC and CLSMC.

Remark 6. In (16), the value of $\alpha$ is designed so that $\hat{\theta}(t)$ is bounded by $\alpha$. That is (16), if $\|\hat{\theta}(t)\| \leq \alpha$, the $\hat{\theta}(t)$ can be updated through

$$
\Omega(\widehat{\theta}(t) t, n \Upsilon q(t))=\Upsilon(t) .
$$

Because $\Upsilon(t)$ is continuous, $\widehat{\theta}(t)$ is also continuous. If $\|\widehat{\theta}(t)\|>\alpha, \widehat{\theta}(t)$ can be updated through

$$
\Omega(\widehat{\theta}(t) t, n \Upsilon q(t))=\Upsilon(t)-\frac{\hat{\theta}(t) \hat{\theta}^{T}(t) \Upsilon(t)}{\|\widehat{\theta}(t)\|^{2}} .
$$

It can be seen from the above equation that $\left(\widehat{\theta}(t) \widehat{\theta}^{T}(t) \Upsilon(t)\right) /\|\widehat{\theta}(t)\|^{2}$ has been increased so that $\|\widehat{\theta}(t)\|$ has been decreased. However, this discontinuity will produce instability phenomenon, in order to avoid this phenomenon, (16) can be simply expressed as (30). In addition, Theorem 2 can also be proved by adaptive law (30):

\section{Simulation Example}

We discuss the following nonlinear systems:

$$
\left\{\begin{array}{l}
\dot{x}_{1}(t)=x_{2}(t) \\
\dot{x}_{2}(t)=x_{3}(t) \\
\dot{x}_{3}(t)=-x_{1}(t)-1.1 x_{2}(t)-0.44 x_{3}(t)+x_{1}^{2}(t)+\sum_{k=1}^{q} u_{k}(t) .
\end{array}\right.
$$

The actuator failures are selected as

$$
\begin{aligned}
& u_{1}(t)= \begin{cases}v_{1}(t)+\phi_{1}(x(t)) \theta, & t<4, \\
-0.8+\phi_{1}(x(t)) \theta, & t \geq 4,\end{cases} \\
& u_{2}(t)= \begin{cases}0.6 v_{2}(t), & t<8, \\
v_{2}(t), & t \geq 8,\end{cases} \\
& u_{3}(t)= \begin{cases}0.5 v_{3}(t), & t<16, \\
v_{3}(t), & t \geq 16,\end{cases}
\end{aligned}
$$

where $\delta_{1}=1, \delta_{2}=\delta_{3}=0, \varrho_{1}=\varrho_{2}=\varrho_{3}=1$ and $c_{11}=1 / 0.5, c_{21}=$ $0.3, c_{31}=0.7, c_{12}=2 / 0.5, c_{22}=0.5$, and $c_{32}=0.3$. The basic functions $\phi_{1}(x(t))=\left[x_{1}(t) x_{2}(t), x_{2}(t) x_{3}(t)\right]^{T}, \phi_{2}(x(t))=$ $[0,0]^{T}, \phi_{3}(x(t))=\left[x_{3}(t), x_{2}(t)\right]^{T}$, and $\theta=[-0.05,0.25]^{T}$. The controller parameters are given as $\emptyset=0.5, \alpha=5, \rho_{1}=\rho_{2}=$ $\rho_{3}=1, \gamma=1, \sigma_{0}=5$, and $b=2$. The desired signal is that when $t \leq 25, x_{d}(t)=[1,0,0]^{T}$, and when $t>25, x_{d}(t)=[0,0,0]^{T}$.

The parameter evaluations for the SMC method and the CLSMC method are shown in Figure 1, and the control inputs and the sliding surface under the two methods are given in Figure 2, and the state variables of $x_{1}(t), x_{2}(t)$, and $x_{3}(t)$ for SMC and CLSMC are shown in Figure 3. It can be seen from the stimulation results that (1) in the SMC method, $u_{2}(t)$ and $u_{3}(t)$ is updated quickly, while in the CLSMC method, $u_{2}(t)$ and $u_{3}(t)$ is updated slowly. (2) The CLSMC method can realize the accurate evaluation of $\theta$ which cannot be realized by the SMC method.

\section{Conclusions}

In this work, the SMC method and the CLSMC method for the SISO nonlinear systems with actuator failures are designed. From the above research, we can draw the following conclusions. (1) The CLSMC method can ensure the convergence of tracking error and accurate parameter evaluation under the PE condition. However, the SMC method can only make the tracking error converge under the IE condition. (2) The CLSMC method has better control capability than the SMC method. The designed of CLSMC method for nonlinear systems with time-varying unknown actuator failures parameter is our future research work.

\section{Data Availability}

All datasets generated for this study are included in the manuscript. 


\section{Conflicts of Interest}

The authors declare that there are no conflicts of interest regarding the publication of this paper.

\section{References}

[1] M. Benbrahim, N. Essounbouli, A. Hamzaoui, and A. Betta, "Adaptive type-2 fuzzy sliding mode controller for SISO nonlinear systems subject to actuator faults," International Journal of Automation and Computing, vol. 10, no. 4, pp. 335-342, 2013.

[2] Y. Li and S. Tong, "Adaptive neural networks decentralized FTC design for nonstrict-feedback nonlinear interconnected large-scale systems against actuator faults," IEEE Transactions on Neural Networks and Learning Systems, vol. 28, no. 11, pp. 2541-2554, 2016.

[3] Y. Li, Z. Ma, and S. Tong, "Adaptive fuzzy output-constrained fault-tolerant control of nonlinear stochastic large-scale systems with actuator faults," IEEE Transactions on Cybernetics, vol. 47, no. 9, pp. 2362-2376, 2017.

[4] S. Tong, B. Huo, and Y. Li, "Observer-based adaptive decentralized fuzzy fault-tolerant control of nonlinear largescale systems with actuator failures," IEEE Transactions on Fuzzy Systems, vol. 22, no. 1, pp. 1-15, 2013.

[5] D. Ye, X. Zhao, and B. Cao, "Distributed adaptive fault-tolerant consensus tracking of multi-agent systems against timevarying actuator faults," IET Control Theory \& Applications, vol. 10, no. 5, pp. 554-563, 2016.

[6] S. Yin, H. Gao, J. Qiu, and O. Kaynak, "Adaptive fault-tolerant control for nonlinear system with unknown control directions based on fuzzy approximation," IEEE Transactions on Systems, Man, and Cybernetics: Systems, vol. 47, no. 8, pp. 1909-1918, 2016.

[7] D. Zhu, Q. Liu, and M. Yan, "An integrated fault-tolerant control for nonlinear systems with multi-fault," International Journal of Innovative Computing, Information and Control, vol. 5, no. 4, pp. 941-950, 2009.

[8] Y. Xu, S. Tong, and Y. Li, "Prescribed performance fuzzy adaptive fault-tolerant control of non-linear systems with actuator faults," IET Control Theory \& Applications, vol. 8, no. 6, pp. 420-431, 2014.

[9] M. Sami and R. J. Patton, "Active fault tolerant control for nonlinear systems with simultaneous actuator and sensor faults," International Journal of Control, Automation and Systems, vol. 11, no. 6, pp. 1149-1161, 2013.

[10] M. Chen and W.-H. Chen, "Sliding mode control for a class of uncertain nonlinear system based on disturbance observer," International Journal of Adaptive Control and Signal Processing, vol. 24, no. 1, pp. 51-64, 2010.

[11] S. Tong and H.-X. Li, "Fuzzy adaptive sliding-mode control for MIMO nonlinear systems," IEEE Transactions on Fuzzy Systems, vol. 11, no. 3, pp. 354-360, 2003.

[12] K.-C. Hsu, W.-Y. Wang, and P.-Z. Lin, "Sliding mode control for uncertain nonlinear systems with multiple inputs containing sector nonlinearities and deadzones," IEEE Transactions on Systems, Man and Cybernetics, Part B (Cybernetics), vol. 34, no. 1, pp. 374-380, 2004.

[13] P. M. Patre, W. MacKunis, M. Johnson, and W. E. Dixon, "Composite adaptive control for Euler-Lagrange systems with additive disturbances," Automatica, vol. 46, no. 1, pp. 140-147, 2010.

[14] X. Wei, N. Chen, and W. Li, "Composite adaptive disturbance observer-based control for a class of nonlinear systems with multisource disturbance," International Journal of Adaptive Control and Signal Processing, vol. 27, no. 3, pp. 199-208, 2013.

[15] H. Liu, Y. Pan, and J. Cao, "Composite learning adaptive dynamic surface control of fractional-order nonlinear systems," IEEE Transactions on Cybernetics, vol. 50, no. 6, p. $2557,2020$.

[16] H. Liu, Y. Pan, J. Cao, H. Wang, and Y. Zhou, "Adaptive neural network backstepping control of fractional-order nonlinear systems with actuator faults," IEEE Transactions on Neural Networks and Learning Systems, vol. 31, no. 12, p. 5166, 2020.

[17] Y. Pan and H. Yu, "Composite learning from adaptive dynamic surface control," IEEE Transactions on Automatic Control, vol. 61, no. 9, pp. 2603-2609, 2016.

[18] Y. Pan, T. Sun, Y. Liu, and H. Yu, "Composite learning from adaptive backstepping neural network control," Neural Networks, vol. 95, pp. 134-142, 2017.

[19] B. Xu and Y. Shou, "Composite learning control of MIMO systems with applications," IEEE Transactions on Industrial Electronics, vol. 65, no. 8, pp. 6414-6424, 2018.

[20] H. Liu, H. Wang, J. Cao, A. Alsaedi, and T. Hayat, "Composite learning adaptive sliding mode control of fractional-order nonlinear systems with actuator faults," Journal of the Franklin Institute, vol. 356, no. 16, pp. 9580-9599, 2019.

[21] H. Sun and L. Guo, "Composite adaptive disturbance observer based control and back-stepping method for nonlinear system with multiple mismatched disturbances," Journal of the Franklin Institute, vol. 351, no. 2, pp. 1027-1041, 2014.

[22] L. Wang, M. V. Basin, H. Li, and R. Lu, "Observer-based composite adaptive fuzzy control for nonstrict-feedback systems with actuator failures," IEEE Transactions on Fuzzy Systems, vol. 26, no. 4, pp. 2336-2347, 2017.

[23] H. Liu, Y. Pan, J. Cao, Y. Zhou, and H. Wang, "Positivity and stability analysis for fractional-order delayed systems: a T-S fuzzy model approach," IEEE Transactions on Fuzzy Systems, vol. $1,2020$.

[24] G. Chowdhary, T. Yucelen, M. Mühlegg, and E. N. Johnson, "Concurrent learning adaptive control of linear systems with exponentially convergent bounds," International Journal of Adaptive Control and Signal Processing, vol. 27, no. 4, pp. 280-301, 2013. 